\title{
Experiencing Moral Distress in Mental Health Nursing in Thailand
}

\author{
Ratchaneekorn UPASEN ${ }^{1}$ and Weeraphol SAENGPANYA ${ }^{2, *}$ \\ ${ }^{1}$ Faculty of Nursing, Chulalongkorn University, Borommaratchachonnanisrisataphat Building, \\ the $11^{\text {th }}$ floor, Bangkok, 10330, Thailand \\ ${ }^{2}$ Faculty of Education, Chulalongkorn University, Phayathai road, Pramingkwaun Building, the $7^{\text {th }}$ floor, \\ Bangkok, 10330, Thailand
}

('Corresponding author's e-mail: weeraphol.s@chula.ac.th)

Received: 16 December 2019, Revised: 12 March 2020, Accepted: 5 April 2020

\begin{abstract}
Mental health nurses have to confront distressing situations in caring for patients and families, in which several predicaments can lead to moral distress. This study explored moral distress experienced by mental health nurses. A qualitative narrative inquiry was used to gain a better understanding of moral distress Together with purposive sampling and snowball methods. The data were collected from 41 mental health nurses who met the inclusion criteria through in-depth interviews and then, the data were analyzed by using content analysis. This narrative study revealed that experiences from keeping the patient safe is a significant core theme among mental health nurses, involving five themes: (1) frustration in giving best work performance, (2) concerning the patient safe, (3) being stressed at work, (4) experiencing self-condemnation, and (5) sleeping difficulty. Support needed to face moral distress was also presented in this study. The stories of moral distress from this study may contribute to the understanding of health professionals concerning how it occurs and what attributes are involved. Situations leading to moral distress and support needed can shed light on the development of policy that can prevent and help relieve moral distress among mental health nurses for a greater quality of healthcare in Thailand.
\end{abstract}

Keywords: Mental health nurses, Mental health nursing, Moral distress, Narrative study

\section{Introduction}

People with mental illness need to receive care and treatment from mental health professionals, particularly from mental health nurses, who play important roles in their healthcare [1]. In caring for individuals with mental illness, however, mental health nurses have to cooperate with a multidisciplinary team. The team is commonly guided by nursing regulations, nursing codes, health policies, and institutional rules to ensure good care for people with mental illness [1]. Mental health nurses often face complex situations, such as overseeing the treatment and care of psychiatric patients who have cognitive, perception, and behavior disturbances. They have to be able to finding better ways to deal with the patient's needs as well as those of the families. In reality, mental health nurses may experience both internal and external obstacles, including insufficient nursing staff, excessive workload, working conditions, lack of supervision, and inadequate training [2]. Furthermore, mental health nurses may confront ethical quandaries known as moral dilemmas while caring for both patients and families $[3,4]$. One consequence of encountering such moral dilemmas is moral distress [2].

There are mental health nurses who have experienced moral dilemmas [4-7]. When such dilemmas go unresolved, it can lead to moral distress. This distress, in turn, impacts the nurses' wellbeing [7]. Moral distress may cause nurses to experience physical and psychological problems, e.g., burnout, intention to leave a position [8], and ineffective working [9]. 
http://wjst.wu.ac.th

Moral distress was first defined by Jameton [10] in the nursing context as a situation that happens when nurses cannot take moral action because of the various barriers despite knowing what morally appropriate actions are needed to protect their patients. There have been studies on the moral distress in healthcare providers in all groups, especially among oncological nurses, emergency nurses, and school nurses. Most of the studies found that nurses experienced moral distress at different levels $[8,9,11,12]$. For example, one study found that nurses and other professionals directly caring for patients experienced significantly higher moral distress than physicians [8]. Previous studies also revealed that the quality of care and patient satisfaction was low when the care providers experienced moral distress. Besides, the moral burden [13], moving from the original workplace to another specialized area, and staff shortages have also been identified [8,9].

However, there is still little research done on moral distress in health professionals, especially among mental health nurses [3,4,6,7]. One quantitative study in Jordan found that 130 mental health nurses have moral distress at a moderately high level [5]. From the study, it was confirmed that mental health nurses experienced moral distress. In Thailand, however, no research has focused on the moral distress occurring in mental health nursing. In addition, how mental health nurses experience moral distress and what situations can lead to moral distress are still insufficiently researched. Understanding the moral distress experienced by mental health nurses is, thus, needed. The knowledge of moral distress in mental health nursing can lead to the development of the quality of care for mental health nursing in Thailand as well as may enable mental health nurses to be aware of moral decision and behave appropriately in their ethical actions.

\section{Materials and methods}

Narrative inquiry was chosen to gain insight into the moral distress of mental health nurses through stories to better understand how they feel and ascertain the specific perspective of the moral situation. The narrative inquiry invites people to tell or write about their experiences and reflections on the situations in storytelling form $[14,15]$.

Table 1 Demographic data of the 41 participants.

\begin{tabular}{|c|c|c|}
\hline Characteristics of participants & $\begin{array}{l}\text { Number } \\
(\mathrm{N}=\mathbf{4 1})\end{array}$ & Percentage \\
\hline \multicolumn{3}{|l|}{ Sex } \\
\hline Male & 7 & 17.07 \\
\hline Female & 34 & 82.92 \\
\hline \multicolumn{3}{|c|}{ Age $($ Max $=52$ years, $\operatorname{Min}=27$ years $)$} \\
\hline 20 - 30 years & 9 & 21.95 \\
\hline $31-40$ years & 21 & 51.21 \\
\hline $41-50$ years & 9 & 21.95 \\
\hline $51-60$ years & 2 & 4.87 \\
\hline \multicolumn{3}{|l|}{ Marital status } \\
\hline Single & 17 & 41.46 \\
\hline Married & 24 & 58.53 \\
\hline \multicolumn{3}{|l|}{ Education Level } \\
\hline Bachelor degree & 21 & 51.21 \\
\hline Master degree & 20 & 48.78 \\
\hline \multicolumn{3}{|l|}{ Length of working with patients } \\
\hline $2-5$ years & 10 & 24.39 \\
\hline $6-9$ years & 10 & 24.39 \\
\hline$\geq 10$ years & 21 & 51.21 \\
\hline$(\operatorname{Max}=21$ years, $\operatorname{Min}=2.5$ years $)$ & & \\
\hline
\end{tabular}


http://wjst.wu.ac.th

\section{Sample selection and participants}

After the research proposal was approved by the research ethics committees, the researchers sent a letter to the directors of the selected psychiatric hospitals to ask permission to collect data. When receiving permission, the researchers met the headward in each ward to coordinate the selection of the participants following the inclusion criteria. Potential participants were recruited using purposive sampling and the snowball technique. The inclusion criteria were: 1) mental health nurses who have been employed in psychiatric hospitals for at least the past 2 years, and 2) the ability to tell of the experience of moral distress, and 3) be willing and able to participate in the research. In this study, the perspectives of mental health nurses were explored through an interview using open-ended questions addressing the experience of moral distress. Saturated data were gained through data collection from 41 mental health nurses with direct experience in caring for patients with mental illness and their families. The participants in this study were 34 females and 7 males. The details are presented in the article of Upasen et al. [16] and also in Table 1

\section{Data collection}

Data collection was performed from November 2017 - September 2018. The data collection took place at the selected psychiatric hospitals in Thailand. The researchers met potential participants, namely, mental health nurses who met the inclusion criteria. These potential participants were invited to participate in the study and share their experiences and reflections on the typology of stories while providing care for people with mental illness. Furthermore, the potential participants were given information about the research processes: the objectives of the study, benefits, and risks of the study, and the right to withdraw from the study without a given reason. Moreover, the researchers informed the potential participants about the confidentiality given for their personal and contact details. Then, the potential participants were given the consent form to sign. After these forms were finished, the $1^{\text {st }}$ author made appointments with the participants to collect data according to day availability. Face-to-face interviews were conducted by the $1^{\text {st }}$ author in Thai to collect the data. The length of the interview was approximately $45 \mathrm{~min}$ for each participant [16]. The interviews were audio-taped and transcribed verbatim. Finally, the researchers also used the snowball technique to further look for participants.

\section{Data analysis}

The transcribed interviews were the data for the narrative research. The data analysis began simultaneously alongside data collection. Data analysis in this research consisted of 2 phases: first, all transcriptions were completed by the researchers. Then, the researchers read the transcriptions carefully with text highlighted relating directly to experiences of moral distress. The transcriptions were reread to identify the narrative features of the highlighted text. A core story "Experiencing moral distress from keeping patients safe" was abstracted from the content of the interviews with plot events put in sequence. All contexts in the psychiatric nursing practice, characters as mental health nurses, patients, health care team members, and families and comments on the action helped enable the reader to understand the story. Direct quotes based on the participants were used in analyzing the narrative data.

During the analysis of the story, the researchers emphasized what had happened and what had not happened, how things were said, what was said, and whether there were any connections in event, time, and sequence. Furthermore, what and how questions were used to gain insights into the story of moral distress: What were the reasons the narrative developed in that way and told in that order? How does she place herself in relation to the audience? How does s/he locate her characters in relation to one another? What was the response of the audience and how did it impact the development of the narrative? Second, the typologies of the story were constructed by the researchers because the types of narratives or stories allowed people to recognize the uniqueness of each story $[15,17]$. Then, the researchers gave a name to the type of stories of moral distress by beginning with the analytic interest. 


\section{The trustworthiness of the data}

Researchers were attentive to maintain the trustworthiness of this qualitative approach. The researchers used 4 criteria by Reissman [14] to enhance trustworthiness in narrative research: 1) persuasiveness: the narratives are convincing readers to read or hear. In this study, the narratives are also supported by the participants' accounts. 2) correspondents: the narratives are recognizable by participants that mean after the researchers found stories of moral distress, the researchers returned stories to validate and confirm meaning from the participants. 3) coherence: in a story, there is an aesthetically consistent relationship among the parts of the narrative; and 4) pragmatic use: the knowledge about moral distress in psychiatric nursing practice can be applied to use to other disciplines.

\section{Ethical considerations}

This research was approved by the Health Ethics Review Board of Chulalongkorn University, reference number $183.1 / 60$, and the 4 psychiatric hospitals before the recruitment of participants. The researchers gave the necessary and complete information for the participants to be comfortable in all the processes of the research. The researchers also took responsibility for protecting the participants by using the key ethical considerations, including informed consent that was proved by the participants. Harms and benefits, confidentiality, and anonymity were employed in all the processes of the study.

\section{Results and discussion}

\section{Experiencing moral distress from keeping the patient safe}

Situations regarding moral distress in mental health nursing practice are associated with the inability to protect the patient's security, both internal and external constraints, which consequently result in the state of "Experiencing moral distress from keeping the patient safe". This situation is considered the core theme of moral distress among mental health nurses who work with patients with mental illness. The 5 themes are found in this study, including the frustration in doing the best, concerning the patient safe, being stressed at work, experiencing self-condemnation, and difficulty sleeping while giving care for patients. Each theme is described as follows:

\section{Frustration in doing the best}

The participants had feelings of frustration in their attempts to do their best for their patients and family. One example of the stories of a participant feeling frustrated was when a patient was trying to commit suicide by trying to hang him/herself and running into a moving car before being admitted to the hospital. After being admitted, the patient was separated into the seclusion room and had to follow the procedure. The participant disagreed with the team members that the patient should not be put there, doubting that the seclusion room was a suitable place for the patient who was able to communicate normally without any signs of mental symptoms. The participant, sometimes, had high expectations of team members that the team members would understand and support the patients like him. One consequence that occurred with the participant related to work stress. The participant then tried to ask himself what was the nurse's role in protecting the patients. In addition, the participant had talked with his family members about what had happened and what he should do for other patients. As one of the participants said:

"Every time, when I restrain a patient who exhibits violent behavior, I give information to the patient about the significance of restraining. Most patients accept and understand what the psychiatric nurses do. Sometimes, I think that I do not want to do this. It is my frustration. It seems that I also don't want to be restrained like the patient. I understand the patient (Case 21, page 1, lines 20 - 23)."

Another participant said he felt frustrated when they provided care for the VIP patients. They felt why they have to give special care to the VIP patients. All patients should treated equally in the same hospital. However, the participant had to provide nursing care for the VIP patient first while other patients had to wait for the nursing treatment, which caused frustration among the participants for this unreasonable responsibility. Since all patients equally receive the same nursing care, there should be fair treatment for all patients. As one participant voiced: 
http://wjst.wu.ac.th

"In my opinion, the healthcare professional codes of conduct or regulations pronounce that we, as nurses, have to provide care for all patients equally. We cannot choose a specific patient for care. I feel frustrated. However, I have to do it. I always ask myself why I have to do (Case 9, page 1, lines 13 - 16)."

\section{Concerning the patient safe}

The participants in the study felt anxiety that stemmed from protection against potential harm to or from patients with violent behaviors. The role and responsibility to protect the patient were deemed essential for the participants. Recounting the stories, one participant informed the researcher that separating patients exhibiting extreme behavior in the seclusion room after being admitted to the hospital was very exasperating. Even though the patients had been treated with special care, they refused to be put in a separate room and even tried to harm themselves. When unexpected, this situation always led to moral distress among the participants and furthered their anxiety. The participant also fears that the situation might recur with other patients. Every time working, the participant tried to keep track of the patient's behaviors to protect against any harm. Notably, there are a few staff on the night shift, and the participant had many patients under their care. However, the participant talked with team members to look for good ways to care for the patients safe. As one participant commented:

"It seems that I did not provide decent care for the patient. I felt fear and anxiety every time I had to separate the violent patient in the seclusion room. I was not happy with the work. I felt anxiety all the time, and I am worried that the same situation might happen again (Case 22, page 1, lines 11 - 15, 20 21).

Another story concerned the referral system. The participant expected that the patient would be treated as holistically as possible by the team members. The participant knew that she could not help the patient, who needed IV fluid or medicine injection. However, the participant only provided bedside nursing care, including adjusting the patient's position appropriately and with warmth. In this situation, the participant was anxious about active treatments for safety. That means that the mental health nurses are worried when they have to use medical equipment, such as using resuscitation machines to save patient's lives. There had been some limitations in the nursing role for taking care of the patient during the transfer. Then, the participant had talked with the headward and team members about what should happen the next time as well as looking for training on physical care to the patients. In the words of the participant:

"Once I had the experience of referring a psychiatric patient to another hospital due to the physical problems of the patient. I could not prescribe treatments like medicine and IV fluid. I could not help the patient in this regard. I knew that the patient needed IV fluid or water, but I could not give it to the patient. The patient's blood pressure began dropping. I just generally had to look after or keep them warm in an appropriate position. I felt a great deal of anxiety that the patient might not be safe (Case 24, page 1, lines 21 - 25)."

\section{Being stressed at work}

Work stress can occur in mental health nursing practice as a result of adverse reactions or the needs of psychiatric patients not meeting treatment requirements including the avoidance of being admitted into the hospital, non-medication adherence, and poor insight. Stories of work stress were described by the participants. Mostly, participants did try to provide good care for patients who do not want to be admitted to the hospital or receive care from health professionals. They had to constrain patients from harming themselves and this resulted in them feeling stress as they were trying hard to guarantee the patient's safety. The nurses knew that the patients displayed aggressive behaviors because of psychotic symptoms. The patients did not intend to behave or act aggressively. The participants understand the patient's symptoms, yet they need to keep the patients and other people safe. Therefore, they need to restrain and separate the patients in the seclusion room, but the patients do not want this. This is exemplified by a participant below:

"I am stressed while taking responsibility in the shifts. There are many psychiatric conditions including depression, delusion, and aggressive behaviors. When the patient's behavior is aggressive, he or she will be restrained by our team. Sometimes, I think that it's not appropriate for patients, for we 
http://wjst.wu.ac.th

could also talk to them to calm down. But I could not tell the team to follow my instructions, so I felt quite stressed (Case 33, page 1, lines 4 - 9)."

Another issue involves the separation of patients with aggressive and criminal behavior in the seclusion room which would be beneficial as special care and protection to themselves and others, as well as the hospital escape. The participants who provided nursing care to patients with aggressive behavior by separating them into separate rooms were also feeling stressful as shared by one of them as:

"In my ward, I looked after the patients who had aggressive behaviors. It is my responsibility to treat them when they were acting violently. I have much stress and have to take care of the patient to be calm while they were having negative emotion and needed to be reduced as well (Case 5, page 1, lines 8 13)."

A narrative is about helping a psychiatric patient living with the family in the community. The participant had an important role to collaborate with these 2 units when the patient was discharged from the hospital. The participant would orientate the patient, family, and community to be ready for the patient to return home. One important issue that may cause stress among the participants is the lack of family and community acceptance of the patient living in the community. The participant had to communicate and convince the family and community about the patient's condition. Trainees are stressed every time they work together with families and communities as said by a participant as follows.

"I was stressed when I collaborated with the patient's family and community after he or she was discharged from the hospital to their community. I understand that the community is still terrified of the patient because they have experienced violent behaviors. I understand well how they feel, and I was stressed as well (Case 25, paragraphs 15 - 18)."

\section{Experiencing self-condemnation}

The experiencing self-condemnation refers to the mental health nurses undergo the feeling of failure to care for patients while they know they should do. Some stories illustrate how the participants feel guilt and what they should do. Most participants shared such experiences which led to their moral distress through a sense of guilt. One participant told a story of following a family request. The related experiences or circumstances involved contacting family members and dealing with miscommunication. The patient had not been admitted to the hospital and this led to the participant feeling guilty. The participant did try to give information to the family members, but the family members still did not understand why the patient was not being admitted. The family members made a complaint to the director of the hospital about their patient not being treated as an in-patient. In the situation, the participants understood the family members and tried to separate the stressed family members in another room. However, in this room the family members felt even more uncomfortable and did not appreciate the experience:

"At first, I felt guilty because I wanted to do my best in the situation, but it was getting worse, so I thought it was my fault. I tried to comfort the family members by making them feel good, so I took them to another room (Case 1, page 3, lines 23 - 25)"

Another narrative is that of a participant's experience with guilt after helping a patient with severe respiratory problems. The patient was physically and mentally aided with the use of medical equipment which was sometimes impossible because of the patient's severe physical health problems and the inadequacy of the equipment in question. The patient then had to be referred to another hospital that night. The patient unexpectedly died on the way before arriving at the hospital. After these events the participant asked herself what had happened and why had the patient died. The nurse knows that such things can happen any time, but still felt guilt for over a month. However, the participant employed strategies to cope with the negative feelings by talking and consulting team members and looking for ways to help the other patients. The participant's moral distress decreased like that of another participant below.

"I feel guilty for a month as it affected my work. When the patient was admitted to the ward, his or her physical symptoms could not be coped with and later the patient had to be sent to a general hospital for the treatment of physical health problems (Case 16, page 2, lines 9 - 11)." 


\section{Sleeping difficulty}

Sleeping difficulty is the last topic covered in this study. As a result of physical health problems, the participants later experience psychological problems including disappointment, anxiety, guilt, and stress after providing psychiatric care. One of the physical problems is the loss of sleep. One of the participants shared stories of when caring for patients with severe physical problems. The participant in the study sought to provide optimal care by monitoring vital signs and keeping the patients safe despite the limitations of medical equipment. However, the patients still experienced severe symptoms and were sent to another hospital. After work, the participants had problems putting themselves to sleep for they were still concerned about the patients' physical symptoms of. As the participants said:

"At night I cannot sleep well. I doze and wake up all night. I am worried about the symptoms of the patient. It's normal for me to take everything seriously. Some nights, I dream about the patient. I always want patients to be in a good condition" (Case 27, page 1, lines 20 - 22).

\section{Support needed to face moral distress}

In this study, the participants mentioned the need concerning administrative support in the form of understanding and listening held annually at the nurses' psychological support center. This kind of support can help participants deal with situations that lead to moral distress. Receiving support on enhancing skills and knowledge, understanding and suggestions are very important issues to help the mental health nurses cope with moral distress to provide quality care to the patients and family members. As one participant said:

"It will be beneficial to nurses if they provide a channel for listening and psychological support. The nurses will have an opportunity to suggest and support needed. If the nurses are ignored to psychological support, they may not be able to provide care for patients effectively" (Case 29, page 6, lines 21 - 23).

\section{Discussion}

The researchers make no claims about the generalization of the findings because of the small sample size and specific setting; however, the findings of this study present evidence that moral distress was experienced even among only 41 psychiatric nurses providing care for patients with mental illness and helping their families. The core theme extracted from this study is "experiencing moral distress from keeping the patient safe". This core theme illustrates that mental health nurses face emotional distress and physical problems following their inability to adequately manage moral dilemmas. These findings are consistent with the study by Musto and Shreiber [18] that found that these nurses experienced moral distress after they tried to keep the patients physically, emotionally, and environmentally safe from harm. Similarly, other studies on the healthcare system found that nurses face moral distress from their practice $[4,5,13,19]$. For instance, 1 prior research has shown that mental health nurses experience moral distress, including feeling squeezed, vulnerable to burnout, distress, feelings of self-doubt, and guilt; feelings of inability and uncertainty in maintaining patients' safety; being in conflict with the mental health agency policy; and the contradictory actions of co-workers [18]. In the previous research, however, there are some different findings with this study since the differences between Thai mental health settings and international mental health settings, including health service system and policy, workforce, and staff training system.

In this study, frustration in doing one's best is one theme identified in this study. That means that the psychiatric nurses did good things for their patients while experiencing feelings of frustration. This finding conforms to the experiences of moral distress among adolescent mental health nursing. These nurses felt uncertain as to how they could keep the adolescent safe. They began to doubt their decisions, nursing roles, and responsibilities in caring for adolescent patients. In addition, they experienced feelings of conflicting values, hearing contradictory messages, and facing obstructed learning $[11,18]$. This finding is also consistent with another study that found 8 mental health nursing instructors expressed their feelings of frustration and powerlessness in psychiatric care [19].

Safe is an important nursing outcome in nursing practice. Thus, keeping patients safe is a crucial role of psychiatric nurses [20]. Mental health nurses provide care and help patients be safe from harm 
arising from psychotic symptoms. According to this study, keeping the patient safe evokes the mental health nurses to experience moral distress: frustration doing the best, concerning harm, selfcondemnation, being stressed in work, and a loss of sleep in this study. These findings are consistent with other studies that found healthcare providers, including nurses, suffered from keeping patients safe as evidenced by their mental health problems through burden, guilt, stress, and feelings of self-doubt $[4,13,21]$. Furthermore, this research revealed that various situations could lead to moral distress including situations of keeping the patient safe, following family requests, cultural awareness, equality of care, and inadequate staff training and inadequate medical equipment.

There are situations regarding to keeping the patient safe such as restraining, separation of the patient, and compulsory treatment. In this study, the participants shared their experiences while providing medical care, the participants are always concerned about the patient's rights and to know what to do to keep the patient safe. Some of these findings are congruent with the results of a study by Heidary et al. [22] that found that these contribute to moral distress, such as medical and treatment errors, and irresponsibility in treatment. In this part, health organization, head nurses, and mental health nurses should provide effective nursing procedures in caring for patients who need intensive care or an active phase.

Following family's request is a situation that leads to moral distress. In care, nurses play an important role in helping the family members of mentally ill patients. The participants provide information and instructions regarding the caring process to the patients or family members, and sometimes they have to deal with some families who want to be involved in every caring process and are too demanding. In addition, they sometimes need to deal with family members who want to leave the patients at the hospital as they are afraid of the patients' behavior, which consequently leads to moral distress for the participants. This finding conforms with the results of a study by Heidary et al. [22] that found that these contribute to moral distress, such as communication problems. The mental health nurses may be continuously trained about communication skills and interpersonal relationships and apply the knowledge in mental health nursing practice effectively.

The situation of cultural awareness can lead to moral distress. The participants are aware of the various cultures and backgrounds of patients while providing nursing care. They are very careful in treating Muslim patients during injection, showering, or dress changing. Some members of the health team pay little attention to these facts but do try to understand the culture and to protect the patients as possible as they can. Since they are not capable to keep things handled, they inevitably experience moral distress. At this point, it is clear that mental health nurses should attention to patient's culture. The mental health nurses also need to learn how to care for patients and families of the patients based on cultural sensitivity [22].

Equality of care situation, in this study, the participants have been exposed to foreign patients and necessary information in different languages needs to be given. However, English language communication is a major problem for the participants because they feel anxious and guilty that the patient may not receive the information as comprehensively as a Thai patient. Irritation may arise from the participants because of concerns about equal care. This is particularly the case for the participants who have experienced providing special care for VIP patients when they believe that all patients should be equally treated. From this finding has been shown that ethical action in nursing care is a very important part [6,7]. Mental health nurses should have awareness and attention about equality of care to all clients. Health organizations as a nursing council, nursing association, and other nursing institutes may set up training projects about an ethical issue in nursing for nurses and other health professionals to enhancing ethical knowledge to a group of health professionals.

Expectations to co-worker's situation, participants work with health team members in caring for the patients and families, including the physician, pharmacist, social workers, and nurse's aide. Sometimes, the participants have high expectations from the co-workers that they will do or follow jobs immediately. Consequently, the participants have experienced moral distress. This finding is found as studies that found that treatment errors, and irresponsibility in treatment, lack of respect from A peer can lead to moral distress [2,22,23,]. At this part can be discussed that teamwork is very important in caring for patients. All health professionals should be aware that caring for patients needs a multidisciplinary team 
http://wjst.wu.ac.th

to reach health outcomes. They should be attended case conference groups and patient round groups to effective plans for patients.

Unforeseen events, mental health nurses have experienced unexpected events such as patients escaping from the hospital, verbal and nonverbal assault from patients, and sudden death. In this study, participants shared their short stories about unforeseen events. Even though they tried to provide quality care, unexpected events still occur. Those contribute to moral distress among the participants. To prevent unexpected events, mental health nurses may have to follow a caring procedure, to keep the patient's symptoms and behavior monitoring. As well, consulting co-workers is needed to care for patients.

Another situation that leads to moral distress is inadequate staff training and resources, the participants in this study have shared their experiences after providing care to the patients under the limitation of having inadequate medical equipment. Also, the limitations of the participants' skill in physical care are viewed as an internal constraint while trying to keep the patient safe from severe physical problems, and the organizational constraints are related to the inability to ensure the patient's safety. Finally, this results in moral distress following the caring process. The findings in this research are also similar to the studies that addressed the causes of moral distress, including organizational barriers, inadequate resources with shortages of staff and inadequate beds, lack of respect from patients, guardians, peers, and bosses [2,23].

Moreover, most of the psychiatric nurses - of all ages - experiencing moral distress needed the understanding and listening provided through the annual event held at the nurses' psychological support center. This finding is consistent with previous studies that have shown similar results - that nurses who experience moral distress used sources of support from peers, teamwork, family members, tried to find solutions, and hospital managers [18,23-25,]. This concurs with the study by Lievrouw et al. [11] that identified 4 coping strategies, namely, thoroughness, autonomy, compromise, and intuitiveness. The strategies make the nurses confront moral distress.

\section{Conclusions}

In mental health nursing practice, several situations can lead to moral distress among mental health care providers. Understanding moral distress is very significant to improve the ability of mental health nurses to cope with the problem. The findings of this study indicated that mental health nurses who face moral distress use various strategies to control it to provide quality care for patients and family members. Mental health nurses should realize moral distress may arise from various situations at any time because they use therapeutic use of self in helping clients. If the mental health nurses have an understanding and awareness of moral distress, they will be able to look for ways to prevent distressing situations from happening.

\section{Recommendations}

Moral distress needs to be controlled at the beginning of the situation to provide quality care. The older mental health nurses who experienced and were able to cope with the moral distress effectively could act as mentors of the younger mental health nurses to help the latter group prevent and cope with moral distress. The mental health nurses may also share barriers in caring to headward to find ways of coping with the problems. At this part, health organizations should provide facilities as staff and/or medical equipment adequacy. As well as clinical reasoning decision and providing quality care will be effectively performed. Moreover, it would be interesting to see future studies explore the coping process or other issues related to moral distress among mental health nurses. Furthermore, the measurement of moral distress in mental health nurses should be developed. Exploring the factors related to or predicting the factors of moral distress should be studied. Moreover, nursing intervention to help mental health nurses cope with moral distress should be developed. 
http://wjst.wu.ac.th

\section{Acknowledgements}

The authors are especially grateful to the psychiatric nurses who participated in this study. This research was supported by the Ratchadaphiseksomphot Endowment Fund of Chulalongkorn University. In addition, the graduate school of Chulalongkorn University is acknowledged for supporting a research assistant (Mrs. Juraipon Samputhanon) to conduct the research.

\section{References}

[1] JC Santos and JR Cutcliffe. European psychiatric nursing/mental health nursing in the $21^{\text {st }}$ century: A person centered evidence-based approach. Cham, Springer, Switzerland, 2018.

[2] N Eren. Nurses' attitudes toward ethical issues in psychiatric inpatient settings. Nurs. Ethics 2014; 21, 359-73.

[3] M Ando and M Kawano. Relationship among moral distress, sense of coherence, and job satisfaction. Nurs. Ethics 2018; 25, 571-9.

[4] S Gabrielsson, S Sävenstedt and M Olsson. Taling personal responsibility: Nurses' and assistant nurses' experiences of good nursing practice in psychiatric inpatient care. Int. J. Ment. Health Nurs. $2016 ; 25,434-43$.

[5] SH Hamaideh. Moral distress and its correlates among mental health nurses in Jordan. Int. J. Ment. Health Nurs. 2014; 23, 33-41.

[6] MH Hem, E Gjerberg, TL Husum and R Pedersen. Ethical challenges when using coercion in mental healthcare: A systematic literature review. Nurs. Ethics 2018; 25, 92-110.

[7] KS Pachkowski. Ethical competence and psychiatric and mental health nursing education. Why? What? How? J. Psychiatr. Ment. Health Nurs. 2018; 25, 60-6.

[8] PB Whitehead, RK Herbertson, AB Hamric, EG Epstein and JM Fisher. Moral distress among healthcare professionals: Report of an institution-wide survey. J. Nurs. Scholarsh. 2015; 47, 117-25.

[9] CL Austin, R Saylor and PJ Finley. Moral distress in physicians and nurses: Impact on professional quality of life and turnover. Psychol. Trauma 2017; 9, 399-406.

[10] A Jameton. Introduction: ethics, nursing, and the crisis in health care. Prentice-Hall, Englewood Cliffs, NJ, 1984.

[11] A Lievrouw, S Vanheule, M Devegugele, M Vos, P Pattyn, V Belle and DD Benoit. Coping with moral distress in oncology practice: Nurse and physician strategies. Oncol. Nurs. Forum 2016; 43, 505-12.

[12] SB Powell, KM Engelke and MS Swanson. Moral distress among school nurses. J. Sch. Nurs. 2018; 34, 390-7.

[13] TL Jansen and I Hanssen. Patient participation: Causing moral distress in psychiatric nursing? Scand. J. Caring Sci. 2017; 31, 388-94.

[14] CK Riessman. Narrative analysis. Sage, Newbury Park, CA, 1993.

[15] CK Riessman. Narrative methods for the human sciences. Sage, London, 2008.

[16] R Upasen, W Saengpanya and J Sambutthanon. Strategies to cope with moral distress among mental health nurses in Thailand. J. Health Sci. Med. Res. 2021; 39, 47-55.

[17] AW Frank. The wounded Storyteller. University of Chicago Press, Chicago, 1995.

[18] L Musto and RS Shreiber. Doing the best I can do: Moral distress in adolescent mental health nursing. Issues Ment. Health Nurs. 2012; 33, 137-44.

[19] B Wojtowicz, B Hagen and CV Daalen-Smith. No place to turn: Nursing students' experiences of moral distress in mental health settings. Int. J. Ment. Health Nurs. 2014; 23, 257-64.

[20] BJ Sadock and AV Sadock. Comprehensive textbook of psychiatry. Lippincott, Williams and Wilkins, Philadelphia, 2017.

[21] R Deady and JM Carthy. A study of the situation, features, and coping mechanisms experiencing moral distress. Perspect. Psychiatr. Care 2010; 46, 209-20.

[22] A Heidary, S Ahrari and NT Chaharsoughi. Moral distress in nursing and its contributors in the context of Iran. Health Spiritual. Med. Ethics 2018; 5, 44-50. 
http://wjst.wu.ac.th

[23] VM Maluwa, J Andre, P Ndebele and E Chilemba. Moral distress in nursing practice in Malawi Nurs. Ethics 2012; 19, 196-207.

[24] C Moe, EI Kvig, B Brinchmann and BS Brinchmann. Working behind the scenes: An ethical view of mental health nursing and first-episode psychosis. Nurs. Ethics 2013; 20, 517-27.

[25] BM Weimand, C Sallstrom, ML Hall-Lord and B Hedelin. Nurses' dilemmas concerning support of relatives in mental health care. Nurs. Ethics 2013; 20, 285-99. 